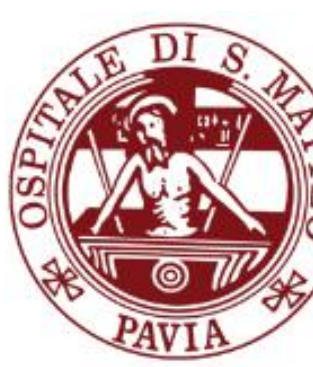

Fondazione IRCCS Mate
Policilinico San Matt
ZR Rogione

\title{
Mechanical CPR with a load distributing band device in out-of-hospital cardiac arrest. An Utstein categories based analysis
}

Sistema Socio Sanitario

Simone Savastano, MD; Enrico Baldi, MD; Alessandra Palo, MD; Maurizio Raimondi,MD; Mirko Belliato,MD; Sara Compagnoni, MD; Fabrizio Canevari,MD; Giorgio lotti,MD; Gaetano M. De Ferrari, MD; Luigi Oltrona Visconti,MD.

Purpose. . In a randomized clinical outcome study of out of hospital cardiac arrest (OHCA), the load distributing banc device (LDB, AutoPulse ${ }^{\circledR}$, Zoll Medical Corporation Chelmsford, MA, USA) did not improve survival to hospita discharge compared to high quality manual CPR. Few studies have explored the effect of the LDB device in standard clinical use with conflicting results. We sought to assess whether the use of the LBD device could affec survival to hospital discharge in the different Utsteir categories.

Methods. All consecutive patients enrolled in our provincia cardiac arrest registry (Pavia CARe) from January 2015 to December 2017 were included and pre-hospital data were computed as well as survival to hospital discharge.

Results:Among 1403 resuscitation attempts the LDB device was used in 235 (18\%) patients. Survival to hospital admission and discharge in the LDB group compared to the manual group was $30 \%$ vs $14 \%(p<0.001)$ and $10 \%$ vs $7 \%$ $(p=0.2)$, respectively. The LDB device was significantly more used for shockable cardiac arrest ( $38 \%$ vs $12 \%, p<0.001)$.

LBD use was a strong independent predictor of survival to hospital discharge for witnessed non-shockable OHCA [ $\mathrm{n}=624 / 1403$, OR $10.5(95 \% \mathrm{Cl} 1.3-82.2) \quad \mathrm{p}=0.028]$. For witnessed shockable cardiac arrest and for non-witnessed and non-shockable cardiac arrest the use of a LDBD was not associated with an increased survival neither to hospita admission nor to hospital discharge.

Conclusions. Utstein categories-based analysis showed that the LDB device positively affect survival to hospital discharge for witnessed non-shockable cardiac arrests but not for shockable arrest and nonwitnessed non-shockable arrests.

\section{RESUSCITATION 2018}

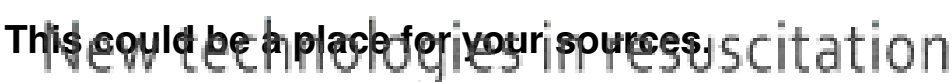
$20-21-22$ September $\bullet$ Bologna $\bullet$ Italy

\begin{tabular}{|r|c|c|c|c|}
\hline \multicolumn{7}{|c|}{ Baseline characteristics } \\
Variable & $\begin{array}{c}\text { Overall } \\
\mathbf{n = 1 4 0 3}\end{array}$ & $\begin{array}{c}\text { LDB device } \\
\mathbf{n = 2 3 5}\end{array}$ & $\begin{array}{c}\text { No LDB device } \\
\mathbf{n = 1 1 6 8}\end{array}$ & $\mathbf{p}$ \\
\hline Male gender (\%) & $844(60)$ & $197(83)$ & $647(55)$ & $<0.001$ \\
\hline Age, median(IQR) (years) & $77(65-85)$ & $63(52.3-71)$ & $80(68-86)$ & $<0.001$ \\
\hline Medical etiology (\%) & $1304(93)$ & $223(95)$ & $1081(93)$ & 0.29 \\
\hline Home location (\%) & $1111(79)$ & $177(75)$ & $934(80)$ & 0.12 \\
\hline EMS witnessed event (\%) & $236(17)$ & $43(18)$ & $193(16)$ & 0.76 \\
\hline Witnessed event (\%) & $1022(73)$ & $203(86)$ & $819(70)$ & $<0.001$ \\
\hline Any bystander CPR (\%) & $472(33.7)$ & $121(51.4)$ & $351(30)$ & $<0.001$ \\
\hline Shockable rhythm (\%) & $260(18.6)$ & $100(42.6)$ & $160(13.7)$ & $<0.001$ \\
\hline EMS response time, median (IQR) & $10.7(8-14)$ & $10.4(7.6-14)$ & $10.8(8-14)$ & 0.53 \\
\hline Resuscitation time, median (IQR) (min) & $27(16-42)$ & $51(36-71)$ & $23.9(15.2-35.6)$ & $<0.001$ \\
\hline ROSC (\%) & $298(21)$ & $95(40)$ & $203(17)$ & $<0.001$ \\
\hline Survived event (\%) & $240(17)$ & $71(30)$ & $169(14)$ & $<0.001$ \\
\hline Survival to hospital discharge (\%) & $106(7)$ & $23(10)$ & $83(7)$ & 0.203 \\
\hline
\end{tabular}

Medical etiology according to Utstein recommendations 2014; EMS response time: from the emergency call to the arrival the first emergency team; Resuscitation time: from the arrival of the first emergency team to the end of ACLS.

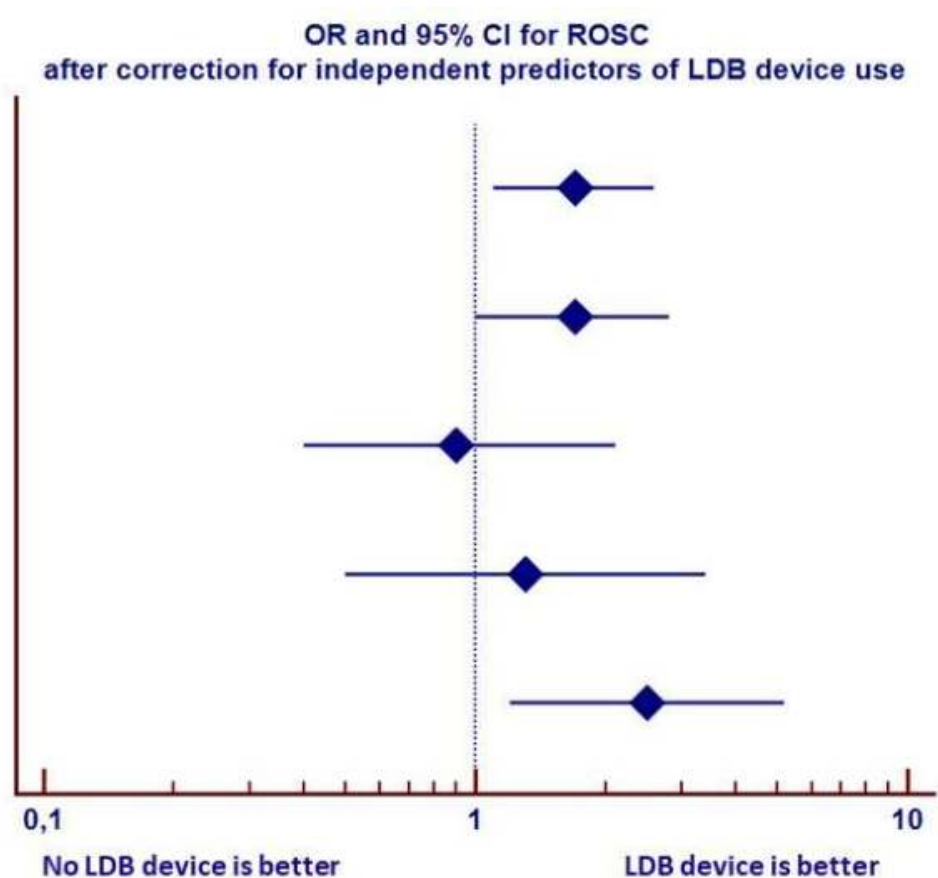

All EMS treated

OR 1.7 (95\%c1 1.1-2.6) $\mathrm{p}=0.02$

EMS witnessed excluded

Shockable bystander witnesse

OR $0.9(95 \%$ ( $10.4-2-2.1) \mathrm{p}=0.82$

Shockable bystander CPR
OR $1.3(95 \%$ C $10.5-3.4) p=0.58$

Non-shockable bystander witn
OR $2,5(95 \% \mathrm{C} 11.2-5.2) \mathrm{p}=0.01$

A

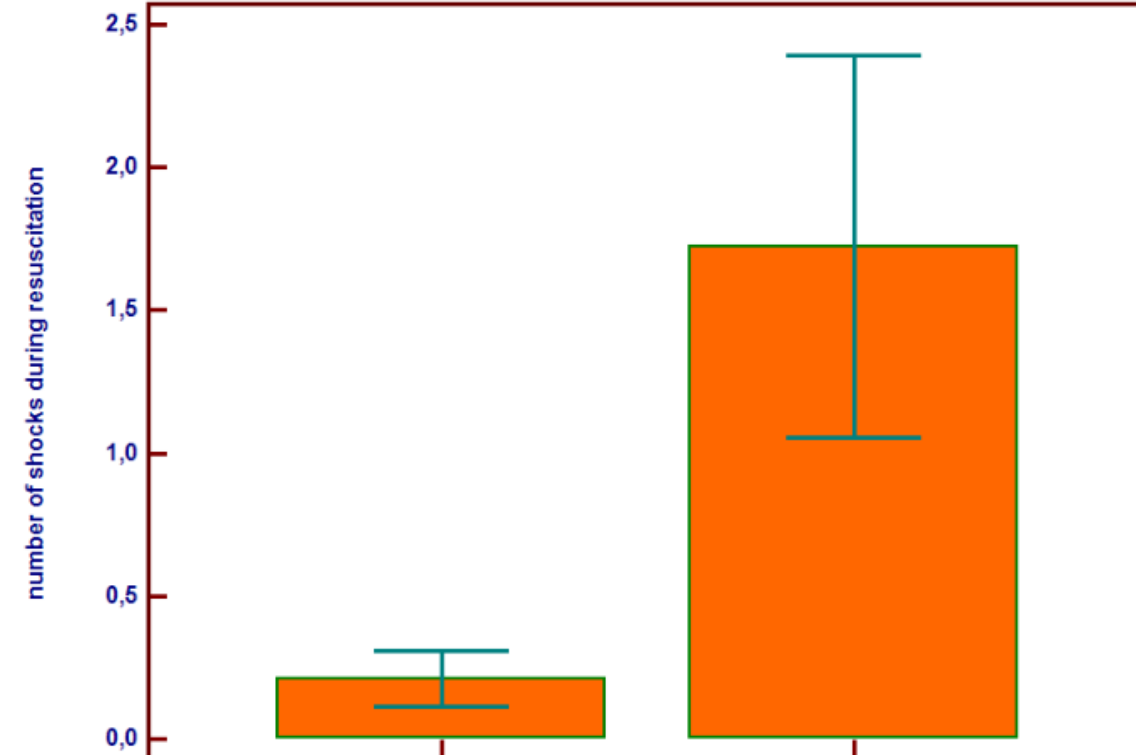

Multivariable logistic regression model for use of LDB device

\begin{tabular}{|l|c|c|c|}
\multicolumn{1}{|c|}{ Variable } & OR & $95 \% \mathrm{Cl}$ & $\mathrm{p}$ \\
\hline Age & 0,97 & $0,96-0,98$ & $<0.001$ \\
\hline Shockable rhythm & 2.7 & $1.8-4$ & $<0.001$ \\
\hline Male gender & 2.3 & $1,5-3,6$ & $<0.001$ \\
\hline Witnessed event & 1.9 & $1.2-3$ & 0.007 \\
\hline Resuscitation time & 1,04 & $1,03-1,05$ & $<0.001$ \\
\hline Bystander CPR & 1.7 & $1.2-2.5$ & 0.003 \\
\hline
\end{tabular}

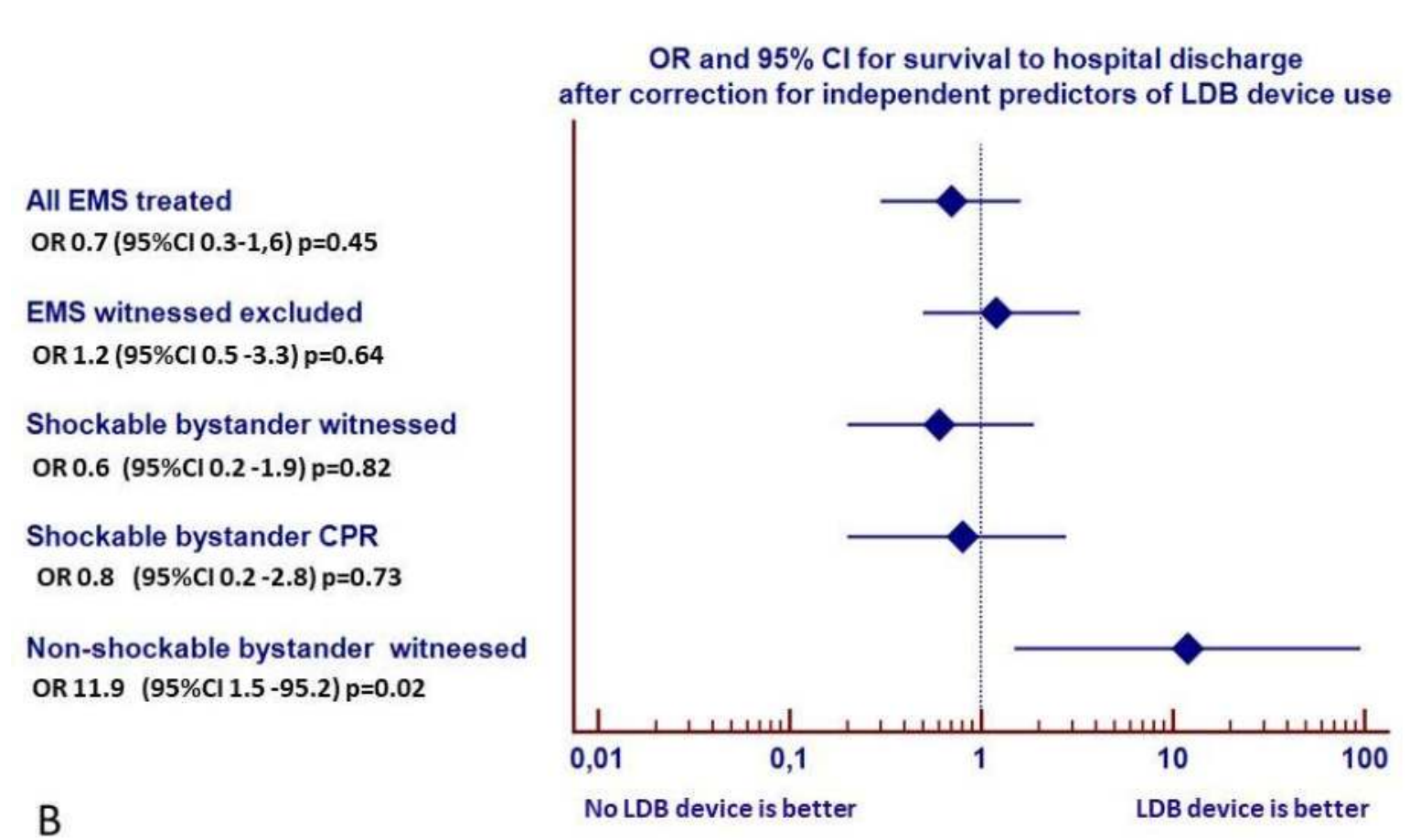

